\title{
DEPOSITION OF COBALT FILMS AND COBALT/ZINC OXIDE MULTILAYERS BY MOCVD
}

P W Haycock, M G Lopez, R W Hardeman, IW Bull, S D Brown, R P Findlay, F Y Ogrin, C C Tang and E W Williams Department of Physics, School of Science and Engineering, Keele University, Keele, Staffordshire, ST5 5BG, UK

\author{
$\mathrm{J}$ Auld and $\mathrm{A} C$ Jones \\ Epichem Ltd, Power Road, Wirral, Merseyside, L62 3QF, UK \\ R J M Griffiths and D Rumsby \\ Metals Research Semiconductors Ltd, Newton Hall, Newton, Cambridge, CB2 5PE, UK
}

\begin{abstract}
Thin layers of cobalt and $\mathrm{Co} / \mathrm{ZnO}$ multilayers have been deposited by metal-organic chemical vapour deposition and characterized by a range of structural and magnetic techniques. In general, the samples exhibited an overall in-plane magnetic anisotropy, the strength of which decreased with decreasing cobalt layer thickness. A buffer layer of $\mathrm{ZnO}$ was found to decrease the strength of the anisotropy with respect to a film of the same thickness deposited onto glass. KEYWORDS: MAGNETIC MULTILAYERS, THIN FILMS, COBALT, MOCVD, ANISOTROPY
\end{abstract}

\section{INTRODUCTION}

Magnetic multilayers show great promise for a wide range of technological applications including magnetooptic (MO) data storage media and magnetoresistive and magnetostrictive sensors. The most favoured systems for MO applications are $\mathrm{Co} / \mathrm{Pt}[1,2]$ and $\mathrm{Co} / \mathrm{Pd}[2]$ multilayer stacks with the individual ultrathin Co layers around $5 \AA$ thick. In this case the multilayers must be hard magnets at room temperature and possess perpendicular magnetic anisotropy. Research into ultrathin Co films on a variety of underlayer materials has shown that the latter can be achieved by use of $\mathrm{Au}[3,4]$, Ir [4], or Ni [4] as well as Pt and $\mathrm{Pd}$. Multilayers of $\mathrm{Co} / \mathrm{W}$ have also been shown to exhibit perpendicular anisotropy [5].

The predominent production technique for magnetic multilayers is sputtering, although molecular beam epitaxy (MBE) has often been used to produce small numbers of high quality samples for research purposes. An alternative technique for production of multilayers is by use of metal-organic chemical vapour deposition (MOCVD). This has been used to good effect for many years in the semiconductor industry for production of III$V$ and II-VI semiconductor multilayer stacks. Deposition of cobalt has previously been reported, as well as iron films from $\mathrm{Fe}(\mathrm{CO})_{5}$ [6]. However, MOCVD has not been used to any great extent for deposition of magnetic multilayer systems. This paper concerns a programme which focused on the development of a viable MOCVD process for the deposition of Co-based multilayers.

We report here the results of investigations into the growth mode and magnetic properties of MOCVD deposited Co thin films and multilayers. As part of this work we have been producing $\mathrm{Co} / \mathrm{ZnO}$ multilayers. This is with a view to evaluating the potential of Co-based metal/dielectric magnetic multilayers, a system which has been proposed to allow the magnetic and optical properties of MO media to be decoupled [7]. However, this kind of multilayer has not yet been extensively investigated experimentally.

The work has been carried out as a collaboration between Epichem Limited, who developed the precursors, Metals Research Semiconductors Limited (MRSL), who designed and built a dedicated MOCVD reactor for the project, and Keele University, where most of the film deposition and evaluation has taken place.

\section{FILM DEPOSITION}

The deposition of cobalt by MOCVD has been carried out previously using a variety of sources, including dicobalt octacarbonyl [8] and cyclopentadienylcobalt dicarbonyl $\left(\mathrm{CpCo}(\mathrm{CO})_{2}\right)[8]$. Zinc oxide deposition has also been reported, from precursors including dimethylzinc tetrahydrofuran adduct (DMZ.THF) with an alcohol [9]. The main task in terms of precursor development was the matching of optimum parameters for the two layers in the stack. We discuss here the results of film deposition using DMZ.THF together with tertiary butanol ( $\mathrm{BuOH})$ for the zinc oxide and $\mathrm{CpCo}(\mathrm{CO})_{2}$ for the cobalt.

All depositions were carried out at atmospheric pressure and a substrate temperature of $350^{\circ} \mathrm{C}$, using hydrogen as the carrier gas. The bubbler temperatures were $12.7,10$ and $17{ }^{\circ} \mathrm{C}$, and the gas flow rates 70,21 and $84 \mathrm{sccm}$ for the $\mathrm{CpCo}(\mathrm{CO})_{2}, \mathrm{DMZ}$.THF and ${ }^{\mathrm{t}} \mathrm{BuOH}$, respectively. The total gas flows to the cell were maintained at $2000 \mathrm{sccm}$ during Co deposition and 329 scem for the $\mathrm{ZnO}$. A vent-run system was employed on the reactor and gas flow switching during multilayer 
deposition was computer controlled, using MRSL software.

\section{FILM PROPERTIES}

Film thickness and crystallographic texture were determined by low-angle and high-angle $x$-ray diffraction respectively, in reflection geometry in-house at Keele and using Station 2.3 of the synchrotron radiation source at the CCLRC Daresbury Laboratory. In addition, transmission high energy electron diffraction (THEED) was performed on specially prepared samples. The microstructure was determined by use of a Hitachi field emission scanning electron microscope (FESEM) and the composition by energy-dispersive $x$-ray analysis (EDS), Auger electron spectroscopy (AES) and Rutherford backscattering (RBS). Magnetic anisotropy was investigated by use of ferromagnetic resonance spectroscopy, magneto-optic magnetometry and vibrating sample magnetometer measurements. The magnetic properties are described in detail elsewhere [10].

The $\mathrm{ZnO}$ films were found to be homogeneous and amorphous for a deposition temperature of $250^{\circ} \mathrm{C}$, with a gradual increase in the number of ellipsoidal crystalline grains with increasing temperature, until highly crystalline films were produced at $350^{\circ} \mathrm{C}$. Although the number of grains increased with temperature, their size remained approximately constant with lengths of 100 $200 \mathrm{~nm}$ and aspect ratios of around 5:1. Figure 1 is an FESEM micrograph of a film deposited at $350^{\circ} \mathrm{C}$.

Films deposited at $350^{\circ} \mathrm{C}$ had a strong preferred orientation with the c-axis normal to the film plane, the only Bragg reflection observed in $\theta / 2 \theta \times$-ray diffraction patterns being the (00.2). Figure 2 gives the high angle diffraction pattern for such a film. These higher temperature depositions $\left(350^{\circ} \mathrm{C}\right)$ led to slightly nonstoichiometric films with a composition of $\mathrm{ZnO}_{0.93}$, as determined by AES. The excess $\mathrm{Zn}$ was in the form of metallic zinc grains with dimensions of around $80 \mathrm{~nm}$. These led to a brown tint. The initial deposition rate at $350^{\circ} \mathrm{C}$ was $18 \AA$ min $^{-1}$, gradually falling to around $1.1 \AA$ $\mathrm{min}^{-1}$ for thicker films, above $220 \AA$.

The $\mathrm{CpCo}(\mathrm{CO})_{2}$ precursor led to deposition of pure $\mathrm{Co}$ at an initial rate of $3.3 \AA \mathrm{min}^{-1}$. No contamination was found from EDS measurements. However, the EDS was not able to detect light elements and RBS was used to confirm that there was no carbon, hydrogen or oxygen present. Thicker films were found to have a uniform granular microstructure (see figure 3) and a close-packed crystal structure with a strong preferred orientation. The close-packed planes were perpendicular to the film plane, even on bare glass substrates, there being only one

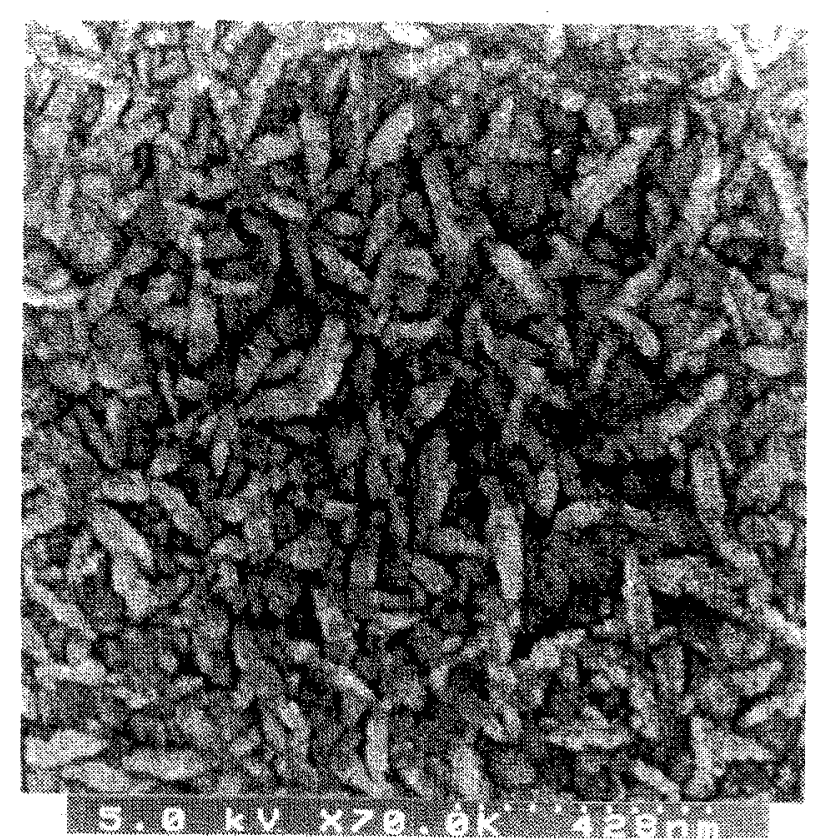

Figure 1 FESEM micrograph of a $\mathrm{ZnO}$ film deposited at $350^{\circ} \mathrm{C}$

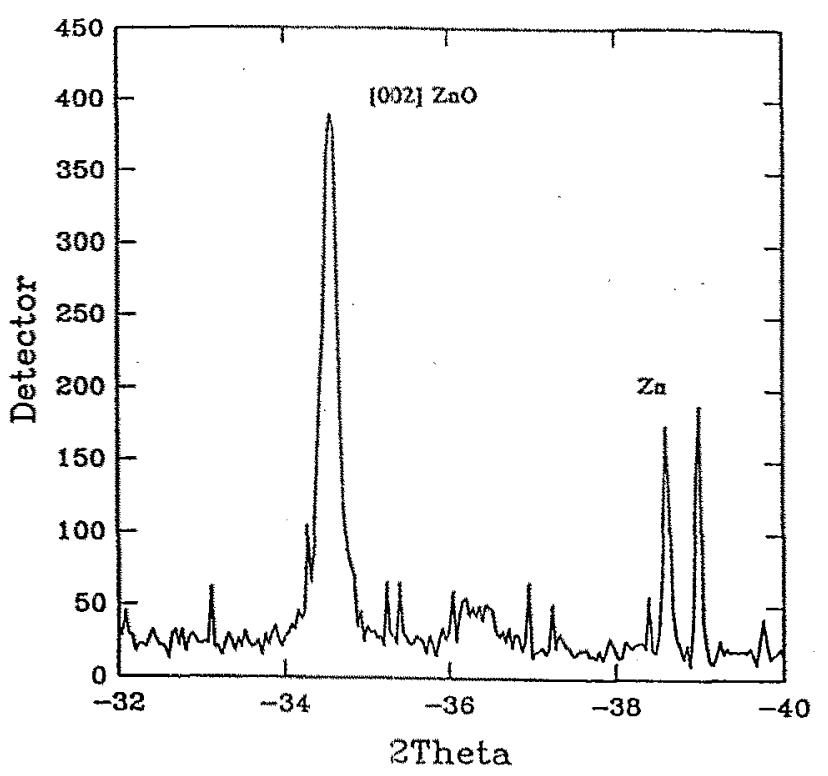

Figure 2 High angle $\mathrm{x}$-ray diffraction pattern from a $\mathrm{ZnO}$ film deposited at $350^{\circ} \mathrm{C}$, showing the $\mathrm{ZnO}(00.2)$ peak and 2 impurity peaks

$\mathrm{ZnO}$ Bragg peak present in the $\theta / 2 \theta \times$-ray diffraction patterns (figure 4). This corresponded to the hcp (00.2) or fcc (111) planes; both phases were found to be present in Co films deposited on carbon grids for THEED analysis, although this may not be representative of the structure of films grown on glass or $\mathrm{ZnO}$. The thinnest films (less than $20 \AA$ ) were found from $x$-ray reflectivity to have an average density of one third that of bulk 


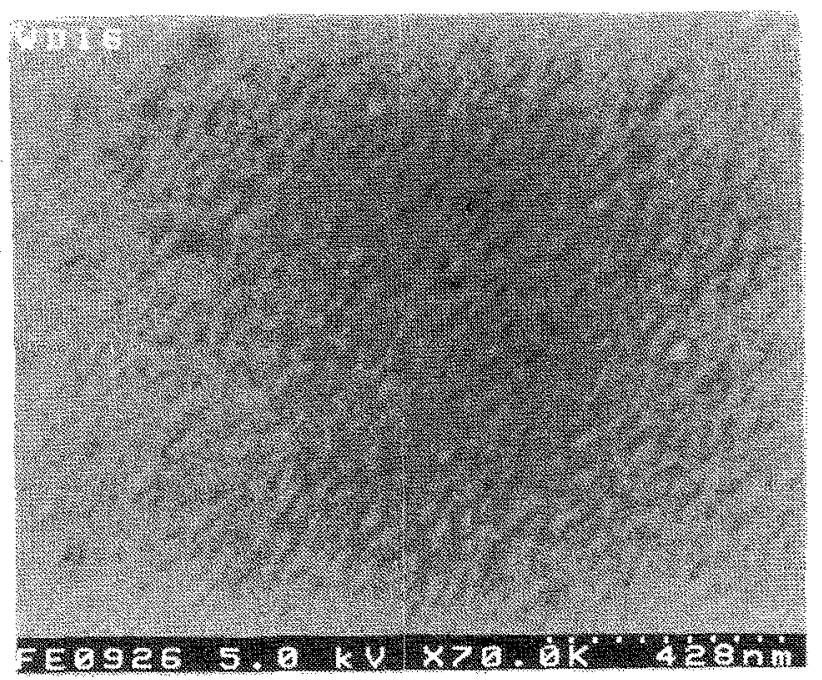

Figure 3 FESEM micrograph of a $250 \AA$ thick Co film.

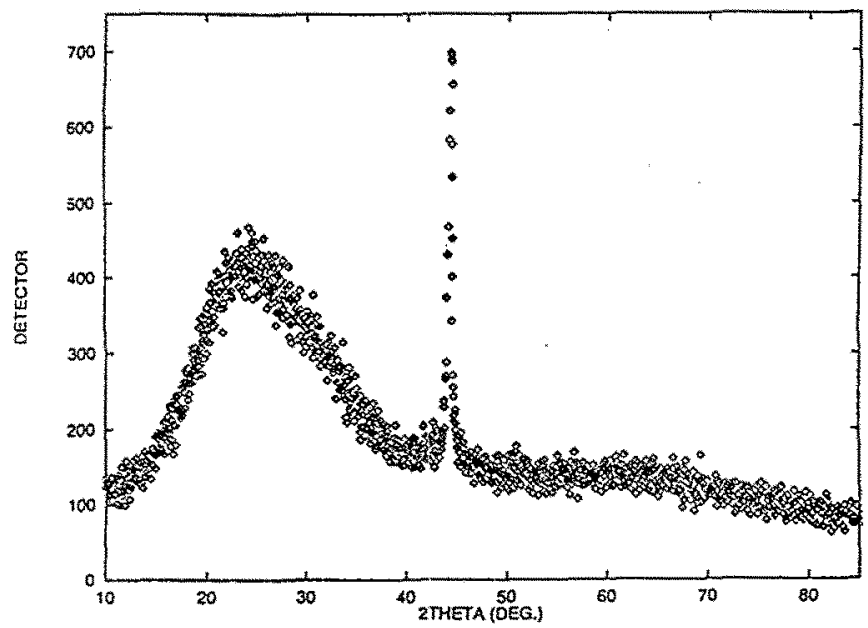

Figure 2 High angle $x$-ray diffraction pattern from a thick $\mathrm{Co}$ film, showing a broad hump from the amorphous substrate and the Co (00.2) Bragg peak

cobalt. This was due to island growth, as determined from FESEM images (figure 5). The island size varied between 20 and $200 \mathrm{~nm}$ depending upon the exact deposition conditions. The overall anisotropy was inplane in all samples, but there was a perpendicular anisotropy field component, the strength of which was inversely proportional to the film thickness [10].

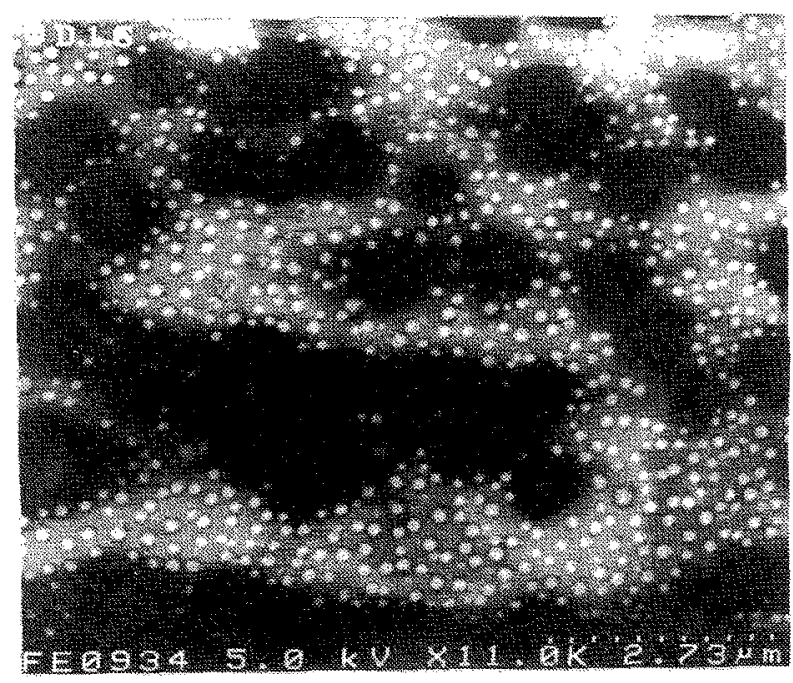

Figure 5 FESEM micrograph of a thin Co film, showing island microstructure

Use of the $\mathrm{CpCo}(\mathrm{CO})_{2}$, for $\mathrm{Co}$ deposition and DMZ.THF with ${ }^{\mathrm{t}} \mathrm{BuOH}$ for the $\mathrm{ZnO}$ has allowed us to produce $\mathrm{Co} / \mathrm{ZnO}$ bilayers and multilayers with minimum mismatch of conditions. We have deposited a range of $\mathrm{Co} / \mathrm{ZnO}$ and $\mathrm{ZnO} / \mathrm{Co}$ bilayers with different thicknesses of both $\mathrm{ZnO}$ and $\mathrm{Co}$. The overall trend is again that the perpendicular magnetic anisotropy component decreases with increasing thickness of the constituent Co layers [10]. However, we have not yet been able to analyse single layers with thicknesses of less than $17 \AA$.

We have deposited $\mathrm{ZnO} / \mathrm{Co}$ multilayers with different thicknesses of $\mathrm{Co}$ and $\mathrm{ZnO}$. The first of these had relatively thick Co layers $(50-200 \AA)$ separated by thin ( $6 \AA$ ) $\mathrm{ZnO}$ layers. This has enabled us to determine the effects of multilayering and use of buffer layers on samples with readily measurable magnetic signals. The magnetic behaviour of the multilayers was similar to that of the single films. One set of optimum conditions involves use of a fairly thin $(\sim 90 \AA) \mathrm{ZnO}$ buffer layer. A sample of the form $\mathrm{ZnO}(90 \AA) /[\mathrm{Co}(50$ $\AA) / \mathrm{ZnO}(6 \AA))]_{10} / \mathrm{ZnO}(90 \AA)$ was found to have good specular optical reflectivity in addition to promising magnetic behaviour. By increasing the DMZ.THF and 'BuOH concentrations in the carrier gas it was found to be possible to produce equally good quality films without a ZnO buffer layer at all. 


\section{DISCUSSION}

We have found that it is possible to produce $\mathrm{Co} / \mathrm{ZnO}$ multilayers with well matched deposition conditions. The fact that the in-plane anisotropy decreases as the films become thinner augers well for the production of films with perpendicular anisotropy. In addition, the optical properties of the multilayers are suitable for MO applications.

We have made use of $\mathrm{ZnO}$ for the buffer layer. This has been used by other workers to induce texture in $\mathrm{Co} / \mathrm{Pt}$ stacks [11]. This approach also avoids the need for a further precursor for a different material. Since it was a (00.2) texture that was found useful as the buffer layer for texturing $\mathrm{Co} / \mathrm{Pt}$ multilayers, in order to attempt to produce perpendicular magnetic anisotropy we have continued to deposit the $\mathrm{ZnO}$ at $350^{\circ} \mathrm{C}$. This may well remain the optimum approach for the buffer layer and the multilayer stacks themselves. The clear amorphous $\mathrm{ZnO}$ films deposited at $250{ }^{\circ} \mathrm{C}$ may ultimately be advantageous for optical overlayers because of their lower optical absorption and scatter.

\section{CONCLUSIONS}

We have successfully produced $\mathrm{Co} / \mathrm{ZnO}$ multilayers by use of MOCVD with well matched deposition conditions for the 2 materials. The magnetic properties are similar to those of sputtered samples. Use of $90 \AA$ thick layers of $\mathrm{ZnO}$ as the buffer and overlayer leads to good magnetic behaviour and reasonable optical properties. Further research is required to obtain overall perpendicular anisotropy and reduced laser scatter.

\section{ACKNOWLEDGEMENTS}

The authors are grateful to Dr R H Jones from the Department of Chemistry at Keele for help with $\mathrm{X}$-ray data collection, Mr S Murray from the Department of Communications and Neuroscience at Keele for help with EDS measurements, and Dr P J Chandler from the School of Mathematical and Physical Sciences at the University of Sussex for RBS data collection. We also wish to thank $\mathrm{Mr}$ A Alexander of Penny \& Giles Ltd for useful discussions. This work was carried out under a LINK programme, funded by the DTI and EPSRC.

\section{REFERENCES}

[1] F J A M Greidanus, W B Zeper, B A J Jacobs, J H M Spruit and P F Carcia: Jap. J Appl Phys 28 (supplement 28-3), 37 (1989)

[2] S Hashimoto and Y Ochiai: J Mag Mag Mater 88, 211 (1990)

[3] J Ferre, G Penissard, C Marliere, D Renard, P Beauvillain and J P Renard: Appl Phys Lett $56,1558(1990)$

[4] Y B Zhang, I A Woollam, Z S Shan, J X Shen and D J Sellmyer: IEEE Trans Mag 30, 4440 (1994)

[5] M Ohkoshi, N Watanabe and K Tsushima: J Mag Mag Mater 113, 92 (1992)

[7] R Atkinson: J Mag Mag Mater 124, 333 (1993)

[6] J V Armstrong, M Enrech, C Decrouez, J G Lunney and J M D Coey: IEEE Trans Mag 26, 1629 (1990)

[8] G J M Dormans, G J B M Meekes and E G J Staring: J Cryst Growth 114, 364 (1991)

[9] T Kaufmann, G Fuchs, M Webert, S Feiske and M Gäckle: Crystallogr Res Technol 24, 269 (1989)

[10] F Y Ogrin, P W Haycock, C Dean and $R$ W Chantrell: these proceedings

[11] S Sumi, Y Teragaki, Y Kusumoto, K Torazawa, S Tsunashima and S Uchiyama: J Mag Mag Mater 126, 590 (1993) 